\title{
Should adult surgical tracheostomies include a Bjork flap?
}

Joshua K. Au MD ${ }^{1}$, Thomas E. Heineman MD ${ }^{1}$, Cecelia E. Schmalbach MD ${ }^{2}$ and Maie A. St. John MD, $\operatorname{PhD} 1,3,4, *$

1. Department of Head and Neck Surgery, University of California-Los Angeles, University of California-Los Angeles Medical Center, Los Angeles, California, U.S.A.

2. Department of Otolaryngology-Head and Neck Surgery, Indiana School of Medicine, Indianapolis, Indiana, U.S.A.

3. Jonsson Comprehensive Cancer Center, University of California-Los Angeles Medical Center, Los Angeles, California, U.S.A.

4. University of California-Los Angeles Head and Neck Cancer Program, University of CaliforniaLos Angeles Medical Center, Los Angeles, California, U.S.A.

Email: Maie A. St. John MD, PhD (mstjohn@mednet.ucla.edu)

*Send correspondence to Maie A. St. John, MD, UCLA Department of Head and Neck Surgery, 10833 Le Conte Avenue, 62-132 CHS, Los Angeles, CA 90095. E-mail:

mstjohn@mednet.ucla.edu

The authors have no funding, financial relationships, or conflicts of interest to disclose.

\section{Background}

Tracheostomy has long been implemented as means of a surgical airway in patients with upper airway obstruction and patients requiring long-term mechanical ventilation support. Temporally, surgical tracheostomy may be performed in an emergent fashion, as in the "slash" tracheostomy, but also as an

This is the author's manuscript of the article published in final edited form as:

Au, J. K., Heineman, T. E., Schmalbach, C. E., John, S., \& A, M. (2017). Should adult surgical tracheostomies include a Bjork flap? The Laryngoscope, 127(3), 535-536. https://doi.org/10.1002/lary.26305 
elective procedure, commonly in patients undergoing resection of the mandible, floor of mouth, pharynx, and larynx.[1] The optimal tracheal incision in surgical tracheostomy has been debated for decades.[1-5] In 1952, following a patient mortality from a displaced cannula, Bjork described creating an inferiorly based tracheal flap through the second, third, and fourth tracheal rings fixed to the skin to secure the tracheostomy lumen, thereafter referred to as the Bjork flap.[2]

Critics of the Bjork flap described the risk of dislodgement of the flap into the tracheal lumen and resultant airway obstruction as the main risk of the Bjork flap tracheostomy.[1] However, various studies have reported the absence of this complication.[1, 3, 5] Additional risks associated with the Bjork flap include tracheal stenosis, tracheal stoma infection, trachea-cutaneous fistula, and dysphagia, which have been cited as potential drawbacks of this technique.[1] In light of these critiques, should adult surgical tracheostomies include a Bjork flap?

\section{Literature Review}

Any definitive recommendation on the use of Bjork flaps in surgical tracheostomies must include two key components: evidence establishing the safety of the Bjork flap as well as a direct comparison of Bjork flap tracheostomies to other surgical tracheostomy techniques to establish a relative benefit. The MEDLINE database was comprehensively searched between time of database inception and March 1, 2016 to include articles of historic importance as well as contemporary reviews.

According to two recent case series by Malata et al. and Hammarfjord et al., the Bjork flap is a safe surgical procedure. Malata et al. reported on 95 Bjork flap tracheostomies performed over a 6-year period in 89 adult patients (aged 17-79 years) undergoing head and neck surgery for malignancy.[1] There were no reported cases of tracheocutaneous fistula, and the median time to stoma closure by secondary intention was 6 days.[1] Additionally, complications of hemorrhage, pneumothorax, displaced tracheostomy, tracheal stenosis, or dysphagia were not reported. The author attributed the absence of tracheal stenosis in this series to the avoidance of overinflation of the cuff, with repeated and accurate 
measurement of cuff pressures. The most common complication was early postoperative chest infection $(n=15)$. Bacterial colonization of the stoma occurred in four patients, with Pseudomonas aeruginosa as the most common species.[1]

In 2015, Hammarfjord et al. reviewed 158 Bjork flap tracheostomies performed in adult patients (aged 18-84 years) undergoing major oral and maxillofacial oncology operations over a 7-year period.[5] The overall complication rate was $5.06 \%$, which is similar to the range of $4.1 \%$ to $11 \%$ in prior heterogeneous series of tracheostomies for head and neck oncology surgery.[5] All complications were minor, including minor bleeding $(n=3)$, tracheocutaneous fistula managed by occlusive dressing $(n=1)$, desaturation resolving with tracheal suction $(\mathrm{n}=1)$, and aspiration pneumonia resolving with antibiotics $(\mathrm{n}=1)$. Patients were decannulated in 2 to 40 days (median = 6 days). No signs of tracheal stenosis were found; however, tracheal stenosis was not evaluated by endoscopy. The absence of tracheal stenosis was attributed to using low-pressure cuffed tubes and downsizing to noncuffed tubes as soon as possible.

Although the Bjork flap appears safe, the evidence surrounding a direct comparison to other surgical tracheostomy techniques is limited. Following the initial description of the inferiorly based Bjork flap,[2] Dukes performed the first retrospective review of 120 patients to compare the rate of tracheal stenosis in the traditional excision-type window tracheostomy to the Bjork flap-based tracheostomy.[3] The degree and level of stenosis was evaluated by bronchoscopy at the time of decannulation as well as at 2 months after decannulation. The incidence of trachea stomal narrowing was noted to be significantly greater in patients with excision window tracheostomy than Bjork flap-type tracheostomies $\left(95 \%\right.$ vs. $10 \%, \chi^{2}=$ 10.42, $P<.01$ ). The authors did not quantify the degree of stomal narrowing. Reduction in tracheal lumen size was noted to be caused by stoma contracture due to scar tissue. The incidence of stricture formation, defined as narrowing to a degree as to cause symptoms, was 3\%. The most common site of stricture was at the distal tracheostomy tube tip in patients on prolonged ventilator therapy (>20 days).[3]

In a prospective cohort study, Lulenski sought to evaluate the degree of tracheal stenosis in 12 adult patients who had Bjork flap tracheostomy compared to four adult patients with vertical incision 
tracheostomy.[4] All patients had their tracheostomy for at least 14 days prior to decannulation. Anteriorposterior and lateral view x-rays of the trachea were performed 4 to 10 months after decannulation. The average anteroposterior and lateral tracheal diameter in Bjork flap patients was $18.0 \mathrm{~mm}$ and $17.2 \mathrm{~mm}$ compared to $16.5 \mathrm{~mm} 14.4 \mathrm{~mm}$ in nonflap patients, respectively. Additionally, the percent loss of lateral diameter at the level of stenosis was 6\% in Bjork flap patients and 27.3\% in nonflap patients. Lulenski concluded that Bjork flap patients experience less postdecannulation tracheal stenosis.

\section{Best Practice}

The available evidence suggests that Bjork flap tracheostomy can be performed with minimal complications. Retropulsion of the Bjork flap causing tracheal obstruction was not reported in any case series. However, despite the clear safety of the Bjork flap and the reported decreased incidence and severity of tracheal stenosis in patients with Bjork flaps compared to incisional or excisional-window tracheostomies, the strength of evidence in the literature is insufficient at this time to recommend the use of Bjork flaps in all adult surgical tracheostomies. Multi-institutional randomized controlled studies of Bjork flap tracheostomies compared to other techniques will be required to conclude that surgical tracheostomies in adults should include a Bjork flap as the standard of care.

\section{Level of Evidence}

Best practice recommendations were made based on an individual prospective cohort study (level 2 evidence),[4] an individual retrospective cohort study,[3] and two case series (level 4 evidence).[1, 5] 


\section{References}

1. Malata C, Foo I, Simpson K, Batchelor A. An audit of Bjork flap tracheostomies in head and neck plastic surgery. Br J Oral Maxillofac Surg 1996;34:42-46.

2. Bjork V. Partial resection of the only remaining lung with the aid of respirator treatment. J Thorac Cardiovasc Surg 1960;39:179-188.

3. Dukes H. Tracheostomy. Thorax 1970;25:573-576.

4. Lulenski G. Long-term Tracheal dimensions after flap tracheostomy. Arch Otolaryngol Head Neck Surg 1981;107:114-116.

5. Hammarfjord O, Ekanayake K, Norton J, Stassen L. Limited dissection and early primary closure of the tracheostomy stoma in head and neck oncology operations: a retrospective study of 158 cases. Int J Oral Maxillofac Surg 2015;44:297-300. 